\title{
Internal dose assessment for the French cohort of uranium workers
}

\author{
Estelle Davesne ${ }^{1, *}$, Olivier Laurent ${ }^{2}$, Eric Samson ${ }^{2}$, Iris Jovanovic ${ }^{3}$, Ségolène Bouet ${ }^{2}$, Cécile \\ Challeton-de Vathaire ${ }^{1}$, and Eric Blanchardon ${ }^{1}$ \\ ${ }^{1}$ Institut de Radioprotection et de Sûreté Nucléaire (IRSN), PSE-Sante/SDOS/LEDI, 92262 Fontenay- \\ aux-Roses Cedex, France \\ ${ }^{2}$ Institut de Radioprotection et de Sûreté Nucléaire (IRSN), PSE-Sante/SESANE/LEPID, 92262 \\ Fontenay-aux-Roses Cedex, France \\ ${ }^{3}$ Amplexor, Montigny-le-Bretonneux, France
}

Recently, several epidemiological studies[1-14] were dedicated to identifying potential health effects from incorporated radionuclides. To do so, the exposure and/or dose of individuals were quantified and associations with health status investigated. Since nuclear workers' health and occupational exposure are carefully monitored, they form a particularly interesting population to study risks potentially induced by internal contamination. To reveal any dose-response relation, lifetime internal dose must be assessed for all workers of the epidemiological cohort.

For this, the measurement results of retained and excreted activity (bioassay) are interpreted with biokinetic and dosimetric models. The models best adapted to the situation are chosen on the basis of known or assumed conditions of exposure: radionuclide(s), isotopic ratios, intake time(s), intake route(s): inhalation, ingestion, wound; physicochemical properties of the radioactive material: solubility and activity median aerodynamic diameter (AMAD) for an aerosol. Some or all of these parameters are, in general, unknown and often difficult to characterize regarding past intakes in which epidemiological studies are interested.

In a subset of the French cohort of workers exposed to uranium during nuclear fuel cycle operations (TRACY, $[3 ; 15]$ ), the exposure conditions are documented by job exposure matrices (JEM) relating workplaces and periods of exposure with chemical forms and isotopic compositions of handled uranium compounds, and by incident registries.

From bioassay data, incident registry, JEM and administrative files, annual absorbed doses received by different organs were estimated for these workers through a dosimetric protocol implemented in the DOSEPI software. DOSEPI allowed the estimation of lifetime doses for more than 2,800 workers in 2.5 hours of computation on a supercomputer, from an Excel ${ }^{\circledR}$ file of bioassay results and exposure conditions translated into input files by Visual Basic ${ }^{\circledR}$ macro procedures.

DOSEPI will be presented in details along with the dosimetric protocol on which it is based. Doses estimated for the TRACY cohort subset will also be presented and the application to other cohorts and exposures will be discussed.

\footnotetext{
*Corresponding author: estelle.davesne@irsn.fr
} 


\section{References}

1. T.V. Azizova, E. Batistatou, E.S. Grigorieva, et al., Radiation Research 189, 371-388 (2018)

2. J.D. Boice, Jr., S.S. Cohen, M.T. Mumma, et al., J Radiol Prot 28, 303-325 (2008)

3. S. Bouet, E. Davesne, E. Samson, et al., International archives of occupational and environmental health (submitted)

4. $\quad$ E.S. Gilbert, M.E. Sokolnikov, D.L. Preston, et al., Radiation research 179, 332-342 (2013)

5. M. Gillies, I. Kuznetsova, M. Sokolnikov, et al., Radiation Research 188, 645-660 (2017)

6. M. Kreuzer, F. Dufey, D. Laurier, et al., International archives of occupational and environmental health 88, 431-441 (2015)

7. M. Kreuzer, C. Sobotzki, N. Fenske, et al., Occupational and environmental medicine 74, 252258 (2017)

8. I.S. Kuznetsova, E.V. Labutina, N. Hunter, PloS one 11, e0162710 (2016)

9. E. Rage, S. Caer-Lorho, D. Drubay, et al., International archives of occupational and environmental health 88, 717-730 (2015)

10. E. Samson, K. Leuraud, E. Rage, et al., Radioprotection (Submitted)

11. J.H. Yiin, J.L. Anderson, R.D. Daniels, et al., American journal of industrial medicine 60, 96108 (2017)

12. L.B. Zablotska, N. Fenske, M. Schnelzer, et al., International archives of occupational and environmental health 91, 91-103 (2018)

13. S. Zhivin, I. Guseva Canu, E. Davesne, et al., Occupational and environmental medicine 75, 270-276 (2018)

14. J. Grellier, W. Atkinson, P. Berard, et al., Epidemiology 28, 675-684 (2017)

15. E. Samson, I. Piot, S. Zhivin, et al., BMJ open 6, e010316 (2016) 